\section{hommes}

\section{Hommes \& migrations}

Revue française de référence sur les dynamiques

migratoires

\section{1 | 2011}

Diasporas sri lankaises

\title{
Les Tamouls de La Chapelle
}

Entre solidarité et dépendance

\section{Philippe Gazagne}

\section{Q OpenEdition}

1 Journals

\section{Édition électronique}

URL : http://journals.openedition.org/hommesmigrations/680

DOI : 10.4000/hommesmigrations.680

ISSN : 2262-3353

Éditeur

Musée national de l'histoire de l'immigration

\section{Édition imprimée}

Date de publication : 1 mai 2011

Pagination : 82-93

ISSN : 1142-852X

\section{Référence électronique}

Philippe Gazagne, «Les Tamouls de La Chapelle», Hommes \& migrations [En ligne], 1291 | 2011, mis en ligne le 31 décembre 2013, consulté le 03 mai 2019. URL : http://journals.openedition.org/ hommesmigrations/680; DOI : 10.4000/hommesmigrations.680 


\title{
Les Tamouls de La Chapelle Entre solidarité et dépendance
}

\author{
Par Philippe Gazagne, \\ docteur en études du développement, chercheur à l'Institut des hautes \\ études internationales et du développement, programme d'étude sur les migrations \\ globales (IHEID, Genève)
}

Les coulisses du "théâtre de guerre". Un acteur se maquille avant d'entrer sur scène. II interprète M. Rajapakse, Ilactuel président sri lankais, La Plaine-Saint-Denis, janvier $2010 \odot$ Vasantha Yogananthan

Le quartier de La Chapelle est un élément clé de la dynamique commerciale et des réseaux sociaux des migrants tamouls vivant en France. Situées en haut du $\mathrm{X}^{\mathrm{e}}$ arrondissement de Paris, ces quelques rues où foisonnent restaurants et entreprises "ethniques" sont au cœur des échanges "communautaires". La fréquentation de ce lieu de socialisation et de partage d'informations est stratégique pour les migrants en quête d'emploi ou de soutien. Néanmoins, ce "territoire tamoul à Paris" est un monde plus complexe que l'on pourrait croire. C'est un microcosme qui en comporte d'autres. 
Un pâté d'immeubles haussmanniens entre deux gares ferroviaires. Leurs rez-dechaussée sont occupés par des enseignes indiennes qui ne laissent guère de place à de rares commerçants non indiens. Le quartier de La Chapelle est constitué de quelques rues en contrebas du boulevard de La Chapelle prises en étau entre la gare du Nord et les voies ferrées menant à la gare de l'Est. La Chapelle est un carrefour, un lieu généreusement desservi où affluent lignes de métro, de RER et de train. Administrativement, il s'agit du quartier Louis-Blanc-Aqueduc. Un peu plus loin, d'autres services et commerces "communautaires", plus parsemés, viennent se greffer au quartier, dans sa périphérie, et profiter ainsi de sa concentration. Certains se font discrets, dans les arrière-cours ou dans des "cagibis" de fortune. Le quartier constitue un point névralgique, un espace marchand, un point de rencontre pour une communauté qui ne réside pas dans ce lieu, éparpillée dans la région parisienne et au-delà. Un passage dans le quartier peut paraître déroutant pour le néophyte. On est vite confronté à une profusion de signes énigmatiques dont un grand nombre échappe à l'entendement de l'autochtone parisien. Les vitrines et les façades sont chargées d'annonces et d'affiches qui s'adressent de toute évidence à une "communauté" linguistique. Si l'écriture latine se décline tant en français qu'en anglais, elle est fortement concurrencée par une calligraphie dravidienne : l'alphabet tamoul est ici prépondérant et permet de diffuser une foule de renseignements insaisissables pour les non-tamoulophones. Sur les affiches et les tracts, seuls quelques caractères latins permettent d'identifier le nom du lieu, la date et l'horaire de l'événement annoncé : manifestation culturelle ou politique, rassemblement ou défilé de protestation, festival de films tamouls, funérailles. Ces messages imprimés, collés sur les murs et les platanes, véhiculent des informations qui traversent les frontières : ce jeune défunt sri lankais dont le cliché inonde le quartier est décédé en Suisse la semaine dernière. D’autres placards renvoient à des événements qui se déroulent en Angleterre ou en Allemagne. Des adhésifs publicitaires plaqués sur les vitrines font la promotion de sites tamoulophones : .com, fr, .uk, .de, .ch, etc. Des petites annonces proposent - à qui peut les déchiffrer - la cession de fonds ou de baux de commerce dans le quartier ou ailleurs dans Paris. Pas de doute, les migrants tamouls occupent ici l'espace. Ils y ont imprimé leurs marques et leurs repères.

\section{La Chapelle, un lieu d'échanges}

Le quartier de La Chapelle, enchevêtrement de quelques artères, est devenu en vingt ans un espace symbolique de la migration sri lankaise en France, qu'il soit désigné comme un "quartier indien" au cceur de Paris ${ }^{(1)}$, comme le "Little Jaffna" de La Chapelle $\mathrm{e}^{(2)}$ ou un "territoire tamoul à Paris ${ }^{(3) "}$. Chacune à sa manière, ces expressions 
évoquent la constitution de cette "centralité commerciale minoritaire (4)" caractérisée par sa densité et le particularisme des commerces. Ce centre d'approvisionnement est indéniablement producteur d'identités communautaires, lieu de reproduction sociale et culturelle apte à perpétuer des usages propres à la société d'origine, à consolider un entre-soi. Cette foule - agrégation de marchands et de consommateurs - semble former un tout cohérent, uniforme.

Néanmoins, La Chapelle n'est pas qu'un espace identitaire, un lieu de rattachement façonné par des migrants hantés par leur consistance collective, exhibant ostensiblement leurs particularismes communautaires. Ces processus - de marquage, d'appropriation ou de territorialisation du quartier - ayant été étudiés ${ }^{(5)}$,je m'intéresse pour ma part à d'autres dynamiques. Il est à mon sens important d'examiner cet espace migratoire comme un lieu non seulement d'inscriptions et de représentations, mais aussi de pratiques et d'interactions, d'initiatives, de fusion et de fragmentation. Dans cette perspective, le quartier est en outre un lieu fonctionnel.

Mon approche des migrations n'est pas guidée par un positionnement identitaire, mais plutôt par l'échange. Il s'agit de considérer cette portion d'arrondissement comme un espace d'interférences sociales, théâtre de stratégies plurielles qui s'entrecroisent, se défient, s'accordent provisoirement ; de dépasser la conception qui considère les communautés migrantes comme des groupes humains intégrés et cohérents,

\section{Le quartier de La Chapelle, enchevêtrement de \\ quelques artères, est devenu en vingt ans un espace symbolique de la migration sri lankaise en France.} pour permettre l'étude d'une pluralité de réseaux entre lesquels il existe de nombreuses passerelles mais aussi une certaine compétition, qui s'accordent et s'opposent, à la fois en contradiction et en complémentarité. Si la dimension communautaire semble importante de prime abord, elle occulte une diversité interne, une pluralité de logiques sociales, de registres identitaires, d'affiliations, de réseaux de solidarité et de stratégies qui tendent à s'articuler autour de différentes composantes : caste, classe sociale, origine régionale, classe d'âge, genre, niveau d'instruction, parcours migratoires, etc.

Passé l'illusion de 1'“appareillage symbolique" mis en place par la "communauté tamoule", je me suis attaché à décrire la complexité des relations en situation de migration. Au-delà de la physionomie de cet espace, il s'agit par conséquent d'aller à la rencontre de ceux qui y transitent (ou qui l'évitent) et de privilégier l'étude des acteurs sociaux et des situations dans lesquelles ils déploient des répertoires de rôles divers. Dans cette posture, nous cherchons à saisir comment cet espace est vécu quotidiennement, en confrontant la permanence du lieu à la sociabilité et à la mobilité de ses circulants. 


\section{Des parcours migratoires en contextes incertains}

À Paris comme à Jaffna ou à Trincomalee, les Sri Lankais agissent à peu de choses près de la même façon : dans des situations caractérisées par l'indétermination, ils mettent en place des stratégies économiques fondées sur des liens sociaux diversifiés. Le parcours des migrants s'effectue dans un contexte d'incertitude à multiples facettes. Cette incertitude concerne tant la réalité de leur parcours que les procédures de demande de l'asile. Leur présence en France est parfois un concours de circonstances. Certains demeurent en France du fait d'un passage avorté vers l'Angleterre. Pour beaucoup, l'indétermination est quasi permanente : ils ne savent pas s'ils vont pouvoir rester ou s'ils devront partir. Cela est notamment lié à la méconnaissance des procédures d'asile, d'ailleurs opaques, voire changeantes ${ }^{(6)}$. Impossible de présager de leurs résultats ni de leur durée. Les requérants vivent une temporalité indéterminée. Certains sont arrivés ici avec l'illusion qu'il était facile d'obtenir des papiers. Bien au contraire. Ils doivent faire avec la précarité de leur statut provisoires, conditionné, en instance de renouvellement ou de confirmation. Leur perspective est celle de leur carte de séjour : temporaire. De la même manière, leurs contrats sont généralement résiliables du jour au lendemain.

Cette indétermination procédurale entraîne une incertitude relationnelle. Pourtant, c'est davantage dans les relations sociales que dans les rapports institutionnels que les migrants vont trouver les ressources nécessaires pour résoudre leurs problèmes. Alors qu'ils font l'expérience d'un statut irrégulier ou provisoire, qu'ils sont confrontés aux codes obscurs de la société et de l'administration françaises, l'échange intracommunautaire leur est indispensable. Dans la réalité, il est à la fois désirable et redoutable. À qui se fier? Comment savoir si l'information donnée est divulguée à bon escient ? Il n'est pas facile de s'assurer de la bonne foi des interlocuteurs ni de l'authenticité ou de la pertinence des informations obtenues.

Alors que son parcours est jalonné de décisions cruciales à prendre dans un laps de temps très court, le migrant est confronté à des comportements et des avis très fluctuants face auxquels il est difficile de faire des choix. Comment se déterminer en fonction du conseil de l'un et de l'expérience de l'autre ? Aux prises avec les subtilités des procédures, confronté à des recommandations contradictoires, qui croire? où se rendre ? à qui confier la traduction de son histoire, le suivi de son dossier ? Répondre à ces questions n'est pas chose aisée, que le migrant soit isolé ou non.

Devant ces incertitudes, les rapports d'interconnaissance fonctionnent comme gage de bonne conduite dans des contextes où les liens sociaux sont prépondérants eu égard à une régulation juridique faible ou insignifiante. De fait, les échanges observés à 
La Chapelle sont faits d'ajustements de pratiques venues d'ailleurs, de savoir-faire éprouvés dans des contextes précaires. Ces rapports d'échange se caractérisent essentiellement par leur insertion dans un ensemble de relations interpersonnelles dont ils sont inséparables analytiquement.

Nombre de migrants qui arrivent en France ne réalisent pas tout à fait un saut vers l'inconnu. Certains ont déjà des parents plus ou moins proches établis en France ${ }^{(7)}$. Ils peuvent compter sur un appui, un accueil au moins provisoire, le temps de trouver un travail, un logement, et de lancer une procédure de demande d'asile. D'autres arrivent en terrain totalement inconnu, comptant éventuellement sur le soutien contingent de quelques pairs côtoyés jadis au village ou à l'occasion d'un travail à Sri Lanka. Nombre d'entre eux sont venus en France parce qu'ils y connaissaient un migrant - plus ou moins proche, parfois distant - qui les y avait précédés. Ils peuvent n'avoir jamais rencontré ces contacts auparavant, recommandés par un parent, un ami de la famille. Sans recours familial, les migrants en appellent à la communauté et tout d'abord aux services proposés par leurs concitoyens pour effectuer les démarches administratives qui jalonnent leur parcours. C'est à La Chapelle qu'ils viendront chercher les appuis les plus divers. Remarquablement, même ceux qui bénéficient d'un entourage familial et donc a priori de recours éclairés, viennent chercher de l'aide ici. Le parcours du demandeur d'asile s'inscrit ainsi dans le tissu communautaire.

\section{Sociabilité et interactions quotidiennes}

La Chapelle constitue à la fois un lieu de ravitaillement et de prestations, un espace de rencontres, un lacis de contacts avec lesquels on échange rumeurs et informations, un vivier d'emplois précaires, un point de collecte, un guichet où l'on vient récupérer des appointements, emprunter une somme d'argent et en envoyer une part à Sri Lanka. Pour ces migrants, il y a maintes raisons de venir à La Chapelle. Untel vient ici faire des photocopies de son dossier à transmettre à l'Ofpra. Un deuxième vient récupérer la traduction d'un papier administratif. Un autre, des photos. Un quatrième veut faire imprimer, puis photocopier un avis de décès. Il tapissera ensuite le quartier de la photo du défunt, annonçant ainsi le moment des funérailles. Un certain Kiru vient payer un caméraman qui l'a aidé le samedi précédent à filmer un mariage de tradition tamoule. Kiru travaille sur les Champs-Élysées, alors que son sous-contractant ceuvre dans les cuisines d'un restaurant japonais du quartier Opéra. Ayant récupéré le cachet, il se déplace ici pour le payer. La Chapelle est une aire centrale où il est 
commode de se retrouver. C'est un point de collecte où les travailleurs nocturnes peuvent livrer les employés diurnes.

Les visites de mes interlocuteurs n'ont rien de régulier. Elles sont au contraire très conjoncturelles. Les passages dans le quartier peuvent se produire par vagues selon les urgences, au rythme des difficultés à régler, par exemple des échéances et des requêtes imposées par l'Ofpra.

Ils viendront alors ici quotidiennement, leur "pedigree" sous le bras, afin de pousser le traducteur à produire les documents en français dans les délais impartis.

Le choix du moment d'une visite n'est en rien anodin. On vient à tel moment pour tenter de rencontrer quelqu'un, une personne en particulier. A contra-

\section{Ici on ne cherche pas} une occupation rémunératrice avec un curriculum vitae et une lettre de motivation. Avec ou sans papiers autorisant le travail, la recherche d'emploi fonctionne par recommandation. rio, on passe en coup de vent dans un créneau horaire qui restreint la probabilité d'une rencontre à éviter. Les passages sont parfois limités au minimum ou étudiés afin d'écarter l'éventualité d'un face-àface avec un créancier ou un rival. Certains m'expliquent qu'ils préfèrent restreindre leurs visites dans le quartier parce qu'ils se voient obligés de se définir, de se positionner par rapport aux débats et aux affiches qui inondent le quartier. S'y rendre implique d'être constamment interpellé sur les questions communautaires. On peut se plaire à venir dans ce quartier tout en redoutant l'hostilité des lieux.

Que ce soit dans la quiétude du matin ou dans la frénésie d'une fin de journée, les échanges et les discussions y vont bon train. À tout moment, toute personne transitant dans le quartier peut difficilement s'affranchir des requêtes des autres. Même les nouveaux arrivés - ceux qui ne peuvent encore compter que sur un maigre réseau social - sont facilement retenus dans leur tournée par d'autres migrants qu'ils n'ont entraperçus qu'une ou deux fois au préalable. À peine salués, ils sont invités à faire un pas de côté pour trouver un semblant de discrétion. L'un cherche un travail, un second veut "louer" des papiers pour travailler, un troisième a besoin d'une "boîte aux lettres". Les uns consultent les autres - notamment les employés en pause, ceux qui ont trouvé un labeur au moins provisoire - pour s'enquérir des besoins de leur patron. Le jeune homme interpellé rétorque qu'il cherche lui-même un travail ou bien qu'il n'a connaissance d'aucune piste. S'il en avait une, il la réserverait à son petit cercle de confiance. Il en serait de même s'il connaissait une personne ressource capable d'aider cet interlocuteur.

Il y en a qui viennent ici tous les jours, dans l'espoir de décrocher une menue besogne ou un à-côté rémunérateur. Devant certains cafés ou gargotes, des jeunes gens semblent 
attendre sans compter, parfois des après-midi entières. Lorsque le patron des lieux obtient une commande pour organiser un banquet à l'occasion d'une des nombreuses festivités qui rythment la sociabilité tamoule, ils peuvent espérer obtenir du travail. Leur disponibilité est alors récompensée. Après une interminable attente et pour une rétribution de 200 euros, le cuisinier s'agitera pendant des heures dans une cuisine huileuse pour livrer le festin le soir même à La Courneuve. Une telle activité implique une flexibilité à toute épreuve, les commandes étant parfois aléatoires ou confirmées à la dernière minute. Certains cafés étonnamment vides réalisent la part essentielle de leur chiffre d'affaires de cette façon. D'une manière très commune, les employés sont payés à la tâche, au gré des besoins.

Ici on ne cherche pas une occupation rémunératrice avec un curriculum vitae et une lettre de motivation. Avec ou sans papiers autorisant le travail, la recherche d'emploi fonctionne par recommandation. Il en est de même pour tout service ou recours : pour obtenir un rendez-vous, pour choisir un prestataire, que ce soit un traducteur, un avocat dans le cadre d'une procédure d'asile, un agent spécialisé dans le transfert d'argent, on y parvient "par l'intermédiaire de", en se présentant comme "le fils de" ou "l'ami de". Ce procédé engage a priori le tiers qui a en principe éprouvé la qualité du pourvoyeur de services et le sérieux du postulant.

\section{Le poids des relations interpersonnelles}

Il est difficile d'évoluer sans s'être reconnu par personne. Dans cette "société" des migrants, il importe de pouvoir compter sur un cercle de connaissances. Sans réseau social, le migrant se trouve aisément mis à l'écart de toute opportunité économique et financière. J'ai pu voir évoluer plusieurs jeunes migrants récemment arrivés, en quête d'un peu de considération "communautaire" et "extracommunautaire". "Ici, ce n'est pas pareil que dans mon village, ces gens ne sont pas les descendants de personnes que je connais", me répètent-ils. Les voilà confrontés à un environnement fort différent, dans lequel ils se trouvent très esseulés. Désorientés par la perte de leurs anciennes références, ils tentent de se constituer un réseau social. Contrairement à ce qu'ils ont vécu à Sri Lanka, ils n'ont ici pour ainsi dire personne pour témoigner en leur faveur. Ils doivent vite engager une procédure de demande d'asile et trouver un travail. Toutefois, cet objectif nécessite d'avoir accès à des informations et à des ressources qui leur seront transmises par l'intermédiaire d'un réseau social qu'il leur faut conjointement bâtir. Dans un contexte incertain, ils posent peu à peu des jalons, apprennent et expérimentent pas à pas diverses relations extra- et intra-communautaires. Ils tentent de se constituer un réseau à même de leur apporter des informations 
fiables et de leur donner les meilleures assurances contre les risques et l'incertitude. La solidarité est fondée sur la réciprocité plutôt que sur la prise en charge. Elle implique un degré de confiance entre individus qui lui-même se construit avec le temps par de lents processus d'apprentissage et de répétition des expériences. Aussi ces jeunes migrants s'appliquent-ils à multiplier les liens, comme pour compenser leur absence de statut. C'est ainsi qu'ils s'attachent à rendre des services à des personnes qu'ils connaissent à peine, à s'engager dans une variété de réseaux sociaux en générant dons et contre-dons. En entretenant une sociabilité produite et reproduite à l'unisson d'échanges alternés de produits et de services, ils cherchent à acquérir du crédit. La concentration commerçante du quartier et les mouvements qu'elle induit sont synonymes de ressources et d'opportunités à saisir. Dans ce nceud communautaire, chacun a ses habitudes, ses fréquentations. Les discussions se font en petit comité. Tout en prenant des nouvelles de la famille et des connaissances communes, on demande de l'aide, on propose des services. On entretient des rapports personnels avec tel ou tel 
commerçant souvent fondés sur des affinités régionales ou villageoises. Ces relations vont bien au-delà des rapports de ravitaillement et d'approvisionnement. Les commerces constituent en quelque sorte des points nodaux de réseaux de solidarité.

\section{Les réseaux sociaux au cœur des commerces}

Mon assiduité dans ces lieux m'a permis de repérer les habitudes d'un certain nombre d'individus et de groupes, rassemblés aux abords de leur(s) commerce(s) de prédilection. Les discussions rassemblent fréquemment des interlocuteurs originaires de la même région ou du même village. Elles se prolongent dans ce coin de Paris par la production et la reproduction de liens sociaux entre gens de même origine géographique. Ici, dans la métropole française, c'est d'abord vers les concitoyens de son village à quelque 8500 kilomètres de là que le migrant sri lankais se tourne pour se trouver soutien et entraide.

Ma Mung ${ }^{(8)}$ qualifie d'"ethnique" le commerce pratiqué par des personnes qui s'appuient sur des réseaux de solidarité communautaire pour l'approvisionnement, le recrutement de personnel, l'achalandage et le financement. À La Chapelle, nombre de gérants enrôlent en priorité voire exclusivement des employés originaires de leur village ou de leur district. Les commerçants font ainsi travailler des individus certes d'une même origine, mais ils leur sont en plus reliés par une chaîne de relations interpersonnelles. Dans le contexte particulier que nous étudions ici, les relations concernées sont avant tout infra-ethniques : cette dimension semble analytiquement plus pertinente que le niveau ethnique. Le recrutement se fait de préférence par connaissances interposées. Cette pratique ne découle donc pas d'un sentiment abstrait de communauté ethnique, ni de la vision d'une solidarité diffuse. Ainsi dans nombre de commerces à La Chapelle, le personnel est majoritairement composé de migrants originaires du même village que le patron. Cet enrôlement de covillageois ou de descendants de gens que les embaucheurs connaissent est considéré à la fois comme une forme de solidarité et comme une assurance : cette interconnaissance est, selon les témoignages, un gage de sûreté, de ponctualité, de discrétion, mais aussi de docilité. En recrutant dans un cercle social de proximité (à l'échelle du village, ces proximités sont tant spatiales que sociales), le gérant produit et reproduit des interactions avec des covillageois. Ces rapports géranttravailleur s'inscrivent dans des rapports de réciprocité. La restriction de l'espace social d'enrôlement permet de s'assurer la coopération des travailleurs en traitant avec un nombre étroit de personnes dont la probabilité de retrouver la trace est forte ${ }^{(9)}$. On confronte le passé pour mieux prévoir le futur. 
En inscrivant les comportements dans des systèmes concrets de relations sociales, ces dispositifs contribuent à l'invisibilité de nombreux usages et pratiques et rendent imperceptibles les jeux de solidarité et de dépendance. De ce fait, les échanges oscillent subtilement entre entraide et subordination, sans être forcément antinomiques.

Les liens observés s'inscrivent dans un ensemble de relations plus ou moins libres ou contraintes caractérisées par un fort contrôle social, comme l'illustre notamment le phénomène de la rumeur (pudinam en tamoul). Phénomène omniprésent à chaque coin de rue de La Chapelle, elle joue indéniablement un rôle central dans la sociabilité tamoule.

\section{Ragots et liens sociaux}

Ici, les nouvelles circulent, se propagent prestement et en permanence. Les propos se colportent de bouche à oreille. Ainsi se répandent les bruits. Par des bavardages en série. Dans la plupart des cas, on n'en connaît pas la source, confuse et diffuse. On se croise dans la rue pour se raconter les histoires en vogue. Les propos concernent des événements survenus dans le quartier, des individus et des membres de la communauté ou encore des liens réels ou supposés entre eux : le cambriolage de la veille dans le soussol d'une bijouterie du quartier ; la visite du préposé d'EDF (Électricité de France) chez le coiffeur d'en face ; le contrôle de sept commerces la semaine précédente ; les déboires financiers d'un boutiquier; les mceurs inconvenantes d'un autre; la fermeture temporaire d'un restaurant ; le voyage en Angleterre ou en Suisse d'un détaillant ; la traîtrise d'un débiteur, etc. On évoque la disparition d'un épicier de La Courneuve : introuvable pendant cinq jours, il vient de réapparaître après avoir honoré sa dette. On se distrait collectivement en partageant les frasques des uns et les travers des autres. Autant de motifs communs de récréation qui permettent de créer et de recréer du lien social. En partageant rires et nouvelles, les acteurs cherchent à entretenir et à renforcer leurs relations et à s'affirmer ainsi au sein de réseaux sociaux. Divulguer scoops et exclusivités permet de se concilier compères et alliés. Chacun s'emploie sans relâche à dégoter des nouvelles, à confirmer ou infirmer tel ou tel racontar. Le plus véloce ou le plus prolifique en la matière se met en valeur : savoir prestement informer ses pairs permet d'accroître son prestige et son statut au sein du groupe.

Le phénomène du qu'en dira-t-on est révélateur d'un antagonisme entre logiques individuelles et contraintes sociales. Si ces bruits qui courent peuvent nous paraître souvent anecdotiques en tant qu'observateur extérieur, ils placent chaque personne face aux conventions imposées par la communauté. Ces ragots remplissent manifestement plusieurs fonctions au niveau collectif. Par ces propos assertifs, la communauté 
exprime les normes du groupe, définit ses frontières, ses critères d'appartenance, qui en fait partie et qui en est exclu, tente de contrôler l'accès ou le passage de personnes extérieures. Ils jouent par conséquent un rôle majeur dans la préservation de la cohésion du groupe, dans l'instauration et la conservation des règles et des conventions. Contradictions et esprit de corps ne semblent cependant pas antinomiques et coexistent de manière ambiguë. Si ces échos collectifs concourent à lier, ils peuvent tout autant exclure et entraîner des sanctions sociales à l'encontre de ceux dont le comportement serait jugé inadéquat.

\section{Conclusion}

Haut lieu de circulation et d'échanges, point nodal de flux migratoires, nous avons contemplé ce quartier de La Chapelle comme une portion d'un vaste espace diasporique. Il s'agissait de fixer notre regard sur ce lieu précis tout en restant sensible à l'existence de liens par-delà les frontières, pour mieux appréhender et décrire les modalités d'entraide économique entre Sri Lankais. Les réseaux de solidarité qui s'y déploient en outre de façon transnationale peuvent aussi être contraignants. Ces quelques pages nous ont amené à complexifier les discours sur les solidarités. Si les migrants cherchent appui dans le groupe d'origine, l'identité communautaire n'est pas un espace d'entraide inéluctable. Au fil de cet article, nous avons souligné l'entrelacs des relations sociales effectives mobilisées par les migrants dans des situations particulières. La solidarité s'inscrit plutôt dans des liens interpersonnels, dans des réseaux sociaux au sein desquels des entraides alternées et des principes de réciprocité coexistent avec des rapports hiérarchiques. Nous avons mis en évidence la manière dont liens de protection et de dépendance s'imbriquent inextricablement dans le quotidien des personnes rencontrées. On observe ainsi un enchevêtrement de logiques opportunistes et solidaires. Entraide et compétition, unité et soumission, réciprocité et endettement, coopération et sujétion ne sont jamais très éloignés.

\section{Bibliographie}

- Axelrod Robert, The Evolution of Cooperation, New York, Basic Penguin Books, 1984.

- Dequirez Gaëlle, "Tamouls sri lankais: le Little Jaffna de La Chapelle”, in Hommes e Migrations, n 1268-1269, 2007, pp. 82-91.

- Fuglerud Oivind, Life on the Outside. The Tamil Diaspora and Long-Distance Nationalism, Londres, Pluto Press, 1999. 
- Gazagne Philippe, "Foreign aid and conflict: post-tsunami fisheries recovery interventions in the war-affected

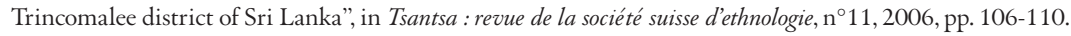

- Gazagne Philippe, "Dettes et migrations : du Sri Lanka à Paris - Modalités d'entraide financière en contextes incertains", thèse de doctorat, Institut des hautes études internationales et du développement, Genève, 2011.

- Glick Schiller Nina, Basch Linda, Blanc-Szanton Christina (dir.), "Towards a transnational perspective on migration: race, class, ethnicity, and nationalism reconsidered", in Annals of The New York Academy of Sciences, vol. 645, 1992.

- Goffman Erving, La Mise en scène de la vie quotidienne, Paris, Minuit, 1973.

- Goreau-Ponceaud Anthony, "La diaspora tamoule: lieux et territoires en Île-de-France", in L'Espace politique, $\mathrm{n}^{\circ} 4,2008$, pp. 21-35.

- Granovetter Mark, "Economic action and social structure: the problem of embeddedness", in American Journal of Sociology, $n^{\circ}$ 91, 1985, pp. 481-510.

- Jones Graham, "Le trésor caché du Quartier indien: esquisse ethnographique d'une centralité minoritaire parisienne", in Revue européenne des migrations internationales, vol. 19 ( $\mathrm{n}^{\circ}$ 1), 2003, pp. 233-243.

- Ma Mung Emmanuel, "L'expansion du commerce ethnique : Asiatiques et Maghrébins en région parisienne", in Revue européenne des migrations internationales, vol. 8 ( $\left.\mathrm{n}^{\circ} 1\right), 1992$, pp. 39-59.

- Marcus George E., "Ethnography in/of the world system: the emergence of multi-sited ethnography", in Annual Review of Anthropology, vol. 24, 1995, pp. 95-117.

- Mary Aude, En territoire tamoul à Paris: un quartier ethnique au métro La Chapelle, Paris, Autrement, 2008.

- Mauss Marcel, "Essai sur le don : forme et raison de l'échange dans les sociétés archaïques", in Sociologie et anthropologie, Paris, PUF, 1983 [1923-1924], pp. 143-279.

- Parry Jonathan, Bloch Maurice, Money and the Morality of Exchange, Cambridge, Cambridge University Press, 1989.

- Raulin Anne, L'ethnique est quotidien: diasporas, marchés et cultures métropolitaines, Paris, L'Harmattan, 2000.

- Servet Jean-Michel, "Le principe de réciprocité chez Karl Polanyi, contribution à la définition de l'économie solidaire", in Revue Tiers Monde, $\mathrm{n}^{\circ}$ 190, 2007, pp. 255-273.

- Vertovec Steven, Transnationalism, Londres, Routledge, 2009.

\section{Notes}

1. Graham Jones, "Le trésor caché du Quartier indien: esquisse ethnographique d'une centralité minoritaire parisienne", in Revue européenne des migrations internationales, vol. 19 (n), 2003, pp. 233-243

2. Gaëlle Dequirez, "Tamouls sri lankais: le Little Jaffna de La Chapelle", in Hommes e Migrations, n 1268-1269, 2007,pp. 82-91.

3. Aude Mary, En territoire tamoul à Paris: un quartier ethnique au métro La Chapelle, Paris, éditions Autrement, 2008.

4. Anne Raulin désigne ainsi une concentration de commerces communautaires territorialement dissociée des lieux de résidence de cette communauté. Le quartier ne compte en effet qu'une faible proportion de résidents tamouls qui demeurent de manière disséminée autour de Paris, essentiellement dans les périphéries nord et est de Paris. Voir Anne Raulin, L'ethnique est quotidien: diasporas, marchés et cultures métropolitaines, Paris, L'Harmattan, 2000, p. 49.

5. Voir notamment Gaëlle Dequirez, op. cit.; Anthony Goreau-Ponceaud, "La diaspora tamoule: lieux et territoires en Île-de-France", in L'Espace politique, n 4, 2008, pp. 21-35 ; Graham Jones, op. cit.; Aude Mary, op. cit.

6. Par exemple, dans la région parisienne, le droit de travailler pendant la procédure de demande d'asile varie selon la préfecture auprès de laquelle le requérant fait sa demande.

7. Voir à ce propos l'article d'Anthony Goreau-Ponceaud dans ce dossier.

8. Emmanuel Ma Mung, "L'expansion du commerce ethnique : Asiatiques et Maghrébins en région parisienne", in Revue européenne des migrations internationales, vol. 8 ( $\mathrm{n}^{\circ}$ 1), 1992, pp. 39-59.

9. Robert Axelrod, The Evolution of Cooperation, New York, Basic Penguin Books, 1984, p. 129. 